\title{
Photoacoustic Cell with Digital Differential Detection
}

\author{
Mariusz Suchenek
}

Received: 6 December 2013 / Accepted: 29 December 2014 / Published online: 22 January 2015

(C) The Author(s) 2015. This article is published with open access at Springerlink.com

\begin{abstract}
Solutions which reduce the impact of the external acoustic noise on the photoacoustic signal rely often on the appropriate modification of the photoacoustic cell structure. The goal is to obtain a frequency response of the cell which suppresses the external noise as much as possible. Another approach is differential detection, in which two microphones are used, and assuming that the external noise signal components from both microphones are identical, their subtraction should result in canceling the external noise. The main difficulty of that solution is that both microphone signal paths should be calibrated to have virtually identical characteristics. The paper presents a differential photoacoustic cell with digital differential detection.
\end{abstract}

Keywords Differential digital detection - Differential photoacoustic cell . Photoacoustic detection

\section{Introduction}

Photoacoustic measurements of a low substance concentration is a big challenge [1,2]. The implementation of such measurements requires the use of a high-power light source and very sensitive detectors. The influence of the external noise can be reduced in such systems by placing the measured specimen into an acoustic cell [3] working at a resonance frequency [4]. Example of such a cell is shown in Fig. 1a.

Additional suppression of the external acoustic noise can be obtained with a differential cell [5]. The use of such a cell has several advantages in comparison to a non-differential configuration. The differential setup allows for subtraction of the

\footnotetext{
M. Suchenek $(\varangle)$

Institute of Electronic Systems, Warsaw University of Technology, Nowowiejska 15/19,

00-665 Warsaw, Poland

e-mail: m.suchenek@ise.pw.edu.pl
} 


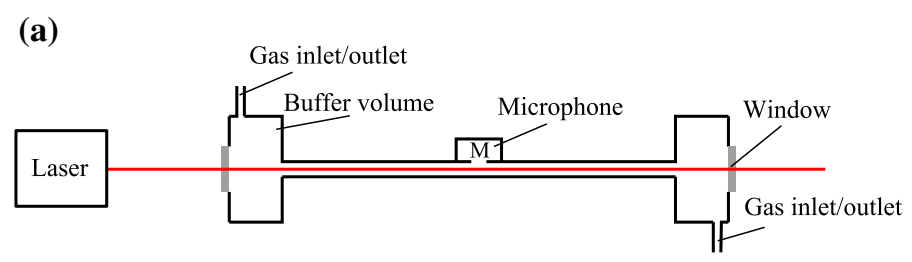

(b)

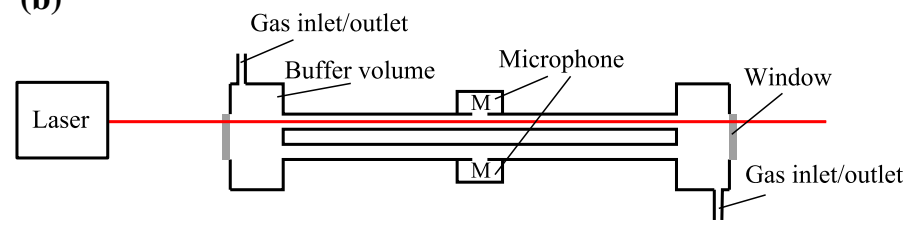

Fig. 1 Example configuration of the photoacoustic cells: (a) non-differential setup and (b) differential setup

noise signal from the photoacoustic signal. The parasitic photoacoustic signal may originate from windows due to the absorption of the light by the windows. Such a parasitic signal is coherent with the excitation signal. In both cases (incoherent noise and coherent parasitic signal), the photoacoustic differential cell is able to significantly improve the signal-to-noise ratio by subtracting external noise from the photoacoustic signal. An example of such a cell configuration is shown in Fig. 1b.

\section{Digital Differential Detection}

To subtract signals from two microphones, analog differential amplifiers are often used [6-8]. A typical electronic circuit performing differential detection in a photoacoustic system [9] is shown in Fig. 2.

Signals from the microphones are amplified independently by preamplifiers in two channels and subtracted from each other by an analog differential amplifier. Both microphones and preamplifiers should be identical, or at least characteristics should be as close as possible to each other. Any non-uniformity in characteristics of the microphones or preamplifiers creates an error during subtraction of the signals from two channels. After the photoacoustic wave is converted to an electric signal and amplified, it is passed through a low-pass filter, working as an anti-aliasing filter and then sampled by an analog-to-digital converter (A/D). Conversion to a digital

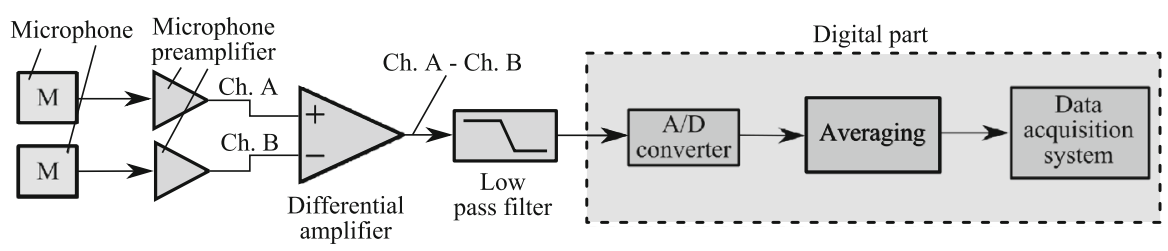

Fig. 2 Block structure of a typical photoacoustic differential detector with analog differential amplifier 


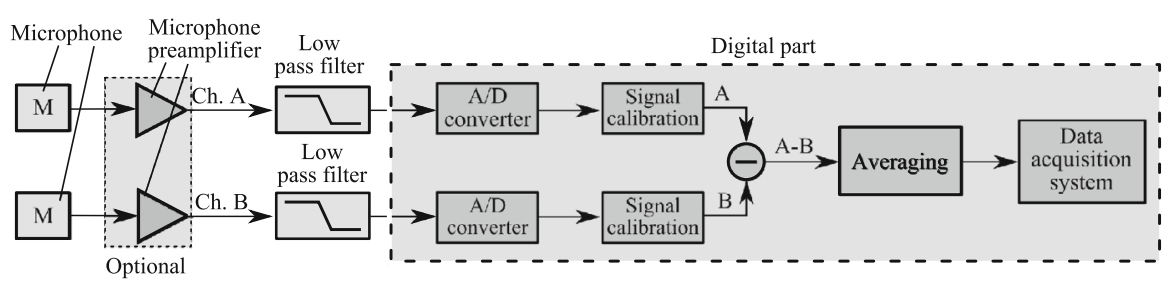

Fig. 3 Block diagram of photoacoustic digital differential detection

domain protects the signal against the noise. Then in a digital system, the signal can be processed according to the user needs. In some experiments instead of the digital block shown in Fig. 2, a lock-in amplifier is used.

In the last few years, significant progress in development of analog-to-digital converters has been observed. Converters are currently available working within an acoustic band with a resolution of 24 or more bits, and the price of these converters is quite reasonable. This allows a design of a fully digital differential amplifier, for which a diagram is shown in Fig. 3.

The input signal in such a system is produced by two independent microphones. Signals from the microphones can be optionally amplified by preamplifiers (if additional amplification is required). Then the signal is band-limited by low-pass filters operating as anti-aliasing filters and converted to a digital domain. After conversion, the signal amplitude and phase from both channels can be independently adjusted according to the calibration.

\section{Experimental Verification}

In order to verify the idea of the digital differential detection, a double acoustic resonator with longitudinal resonance, similar to that presented in Fig. 1b, was designed. The cell prototype was made of plexiglass. A photo of the cell is presented in Fig. 4. The diameter of each duct is $6.5 \mathrm{~mm}$, and the length is $79 \mathrm{~mm}$. The first resonance frequency of the cell was measured at $2.3 \mathrm{kHz}$.

An acoustic signal was induced in the cell by laser stimulation at a frequency of $2.25 \mathrm{kHz}$. The measured gas was $\mathrm{NO}_{2}$, excited at $447 \mathrm{~nm}$. In all conducted measurements, signals from the microphones were recorded by the analog-to-digital converters together with a signal from the calibrated analog differential amplifier and then analyzed offline. A comparison between obtained measured data from the analog differential amplifier and digitally subtracted signal is presented in Fig. 5.

From the series of measurements, the first harmonic was calculated. The first 30 measurements correspond to the signal without the laser stimulation; thus, the recorded data represent background noise. Further measurements correspond to the photoacoustic signal resulting from the gas. Absorption results of both methods are practically identical, in the part of the data which correspond to the background noise as well as in the part where laser stimulation was applied. 

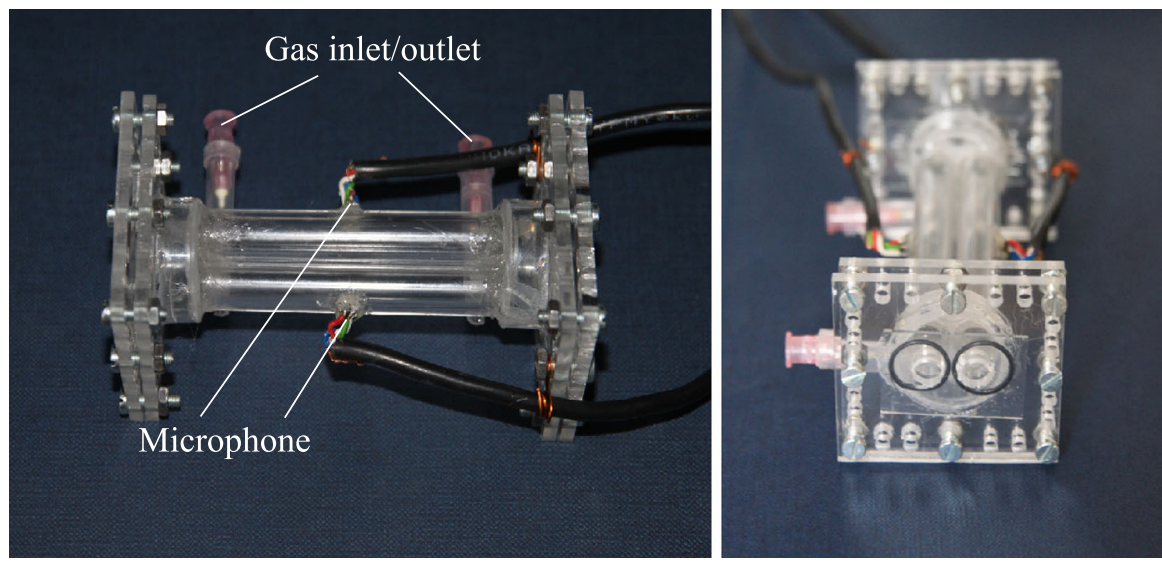

Fig. 4 Photo of the photoacoustic cell used in measurements

Fig. 5 Measured signal from analog differential amplifier and digitally subtracted signal

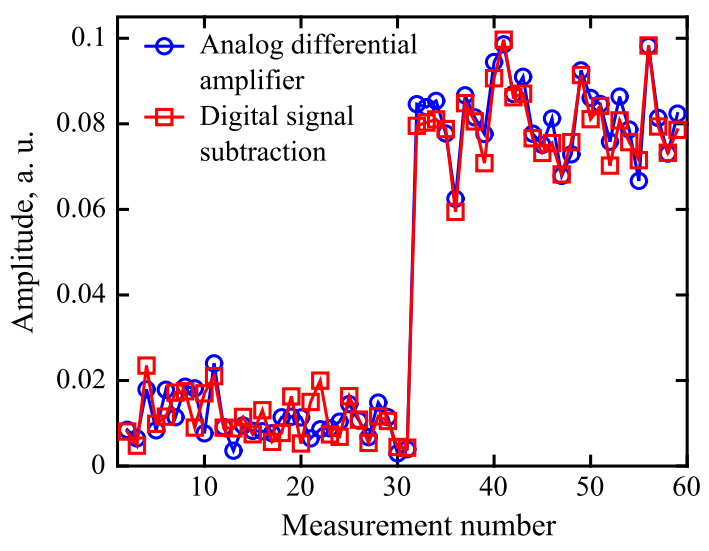

\section{Conclusions}

This paper presents a new approach to photoacoustic differential signal detection. Unlike in the standard approach relying on a differential amplifier, in the present solution, the signals from the microphones are converted to a digital domain and then calibrated and subtracted. The results obtained from the digital subtraction are consistent with results from the analog differential amplifier. Signal calibration can be performed in the software in the digital system, with no change in the analog circuits. Correction factors can be applied in the digital domain which is much simpler to perform than in the analog circuit. This enables precise correction and reduces the impact of non-uniformities of the microphones and the whole analog front end.

Open Access This article is distributed under the terms of the Creative Commons Attribution License which permits any use, distribution, and reproduction in any medium, provided the original author(s) and the source are credited. 


\section{References}

1. J.P. Besson, S. Schilt, L. Thevenaz, Spectrochim. Acta A 63, 899 (2006)

2. C.K.N. Patel, M. Pushkarsky, A. Tsekoun, I.G. Dunayevskiy, R. Go, Proc. Natl. Acad. Sci. 103, 10846 (2006)

3. A. Miklos, P. Hess, Rev. Sci. Instrum. 72, 1937 (2001)

4. J.M. Rey, M.W. Sigrist, Sens. Actuators. B 135, 161 (2008)

5. A. Elia, P.M. Lugar, C. Di Franco, V. Spagnolo, Sensors 9, 9616 (2009)

6. V.A. Kapitanov, A.M. Solodov, T.M. Petrova, Y.N. Ponomarev, Int. J. Spectrosc. 2010, 1 (2010)

7. V. Zeeninari, B. Parvitte, D. Courtois, V.A. Kapitanov, Y.N. Ponomarev, Infrared Phys. Technol. 44, 253 (2003)

8. V.A. Kapitanov, Y.N. Ponomarev, I.S. Tyryshkin, A.P. Rostov, Spectrochim. Acta A 66, 811 (2007)

9. A.V.R. Kumar, Application of Laser Induced Photoacoustic Effect for the Study of Gases and Solids, Ph.D. Thesis, Cochin University of Science and Technology, Cochin, India, 1992 